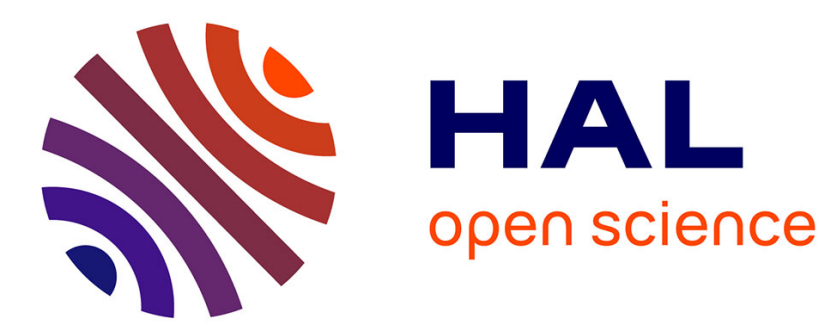

\title{
ULTRASONIC RESPONSE TO TWO AND FOUR LEVEL QUANTUM SYSTEMS
}

\author{
A. Granato, K. Hultman, K.-F. Huang
}

\section{To cite this version:}

A. Granato, K. Hultman, K.-F. Huang. ULTRASONIC RESPONSE TO TWO AND FOUR LEVEL QUANTUM SYSTEMS. Journal de Physique Colloques, 1985, 46 (C10), pp.C10-23-C10-26. 10.1051/jphyscol:19851004 . jpa-00225292

\section{HAL Id: jpa-00225292 https://hal.science/jpa-00225292}

Submitted on 1 Jan 1985

HAL is a multi-disciplinary open access archive for the deposit and dissemination of scientific research documents, whether they are published or not. The documents may come from teaching and research institutions in France or abroad, or from public or private research centers.
L'archive ouverte pluridisciplinaire HAL, est destinée au dépôt et à la diffusion de documents scientifiques de niveau recherche, publiés ou non, émanant des établissements d'enseignement et de recherche français ou étrangers, des laboratoires publics ou privés. 


\author{
A.V. GRANATO, K.L. HULTMAN ${ }^{+}$AND K. -F. HUANG ${ }^{+}$ \\ Department of Physics and Materials Research Laboratory, \\ University of IIIinois at Urbana-Champaign, Urbana, \\ IIIinois 61801, U.S.A.
}

\begin{abstract}
The theory already available for the ultrasonic response to a quantum twolevel system for amorphous materials 18 discussed and adapted to two and four level systems in crystals. It is given in a simple physical way which helps make clear the distinction between resonance and relaxation. Resonance and no relaxation occurs for small enough static strain, while for large enough strain, the opposite is true. For a TLS, relaxation occurs by a direct process, with a rate inear in temperature in the measurable range. This leads to a Debye response, but the temperature dependence is inverted from that usually found for faster relaxation rates. At low enough temperatures the relaxation strength deviates from the classical $1 / T$ behavior, going to zero as the upper state becomes depopulated, whlle the resonance strength saturates. At high temperatures, the sum of these approaches the classical value. Relaxation and resonance strengths are given for two and four level systems as a function of temperature and strain for two strain distributions.
\end{abstract}

The theory of ultrasonic response to a two-level system (TLS) for amorphous materials taking account of distributions of internal strain and barrier heights is well developed and familiar $[1,2]$. Oddly enough, the theory for the simpler TLS applying to defects in crystals requiring only strain distributions seems to be less well famlliar. For example, it is not generally appreciated that elastic constant relaxacion due to tunneling between equivalent sites in the absence of strain (8ymmetric TLS) cannot occur.[3-5] The elastic constant change $\&$ is by definition given as

$$
\partial C=\partial^{2} F(\varepsilon) / \partial \varepsilon^{2}
$$

where $F$ is the free energy per unlt volume, $\varepsilon$ is an elastlc stratn, and $C$ is the elastc constant belonging to that strain. Strictly speaking, $C$ and $\varepsilon$ are tensor quantities. In what follows we suppose for simplification that only one strain component is effective. The free energy is given by

$$
F=-f k T \ln 2
$$

where $\mathrm{Z}$ is the partition function given by

$$
Z=\Sigma_{1} \exp \left[-\left(E_{1} / k T\right)\right]
$$

where $E_{1}(\varepsilon)$ are the states of the system, and $f 18$ the number of defects. There are two $k$ inds of effects resulting for dipolar point defects. These are parelastic and dielastic [6-9], corresponding to magnetic dipolar effects. These can be illustrated with the simple two-level system shown schematically in Fig. 1. For $\epsilon=0$, the dipole is supposed to have two equivalent ortentations. The ground state is tunnel-split with a gap of $24_{0}$. If a stress or strain $\varepsilon$ is applied, one orientation become energetically favored. For fintte strain the gap $2 \Delta$ is given by

$$
\Delta=\left(\Delta_{0}^{2}+\alpha^{2} \varepsilon^{2}\right)^{1 / 2}
$$

where a 18 atraln coupling constant. If time is avallable, transitions will occur, bringing the system back Into thermal equilibrium.

For $T<\Delta$, only the ground state ls populated. For small ultrasonic stress amplitudes, the elastic constant is given by the curvature of the ground state. The dipole moment is Induced, proportional to the strain $\varepsilon$, and the change 18 called dielastic. This response has a resonance-like character. The resonance frequency ls normally far above the MHz range. Present addresses: (a) Geomation Corp., Wheat Ridge, Colorado

(b) National Chiao University, Hisinchu, Taiwan 

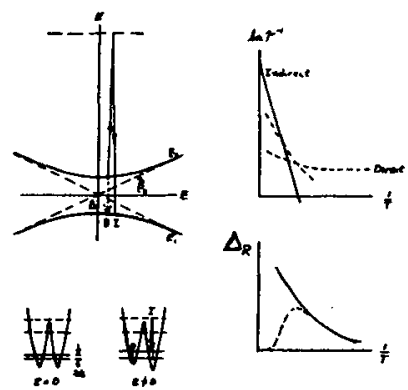

Fig. 1. Schematic illustration of potential, energy levels, transition processes, transition rates and relaxation strength $\Delta_{R}$ for a two level $8 y 8 \mathrm{tem}$, or a four level system Including dashed lines for $B$. Classically $\Delta_{0}=0, \tau^{-1}$ is Arrenhentus, and $\Delta_{R} \propto 1 / T$
As a result no attenuation is seen, but only the low frequency change of elastic constant is found. We restrict our selves in what follows to the case of hux< $\Delta_{0}$, where $\omega$ is the ultrasonic frequency. We call $\delta / C$ the resonance strength 4 , which is then given by taking $F=-f \Delta(\varepsilon)$ to obtain

$$
\Delta_{S}=\delta C / C=-\left(f \alpha^{2} \Delta_{0}^{2}\right) /\left[C\left(\Delta_{0}^{2}+\alpha^{2} \varepsilon^{2}\right)^{3 / 2}\right]
$$

At findte temperature, the upper level becomes populated. It has opposite curvature, leading to a reduction of $\mathrm{Ag}_{\mathrm{g}}$ with increasing temperature.

At finite temperature phonon induced transitions become possible. For the two level system shown, however, no relaxaation takes place for small amplitude ultrasonic waves about $\varepsilon=0$ because the energy of the states is flat for small $\varepsilon$, maintaining equilibrium population of the states. For $\Delta_{0}=$ 0 , the states are given by the dashed lines. This can represent a classical Snoek system, for example Fe-C, (If one of the levels is doubly degenerate). Now if a strain is applied, equilibriun is disturbed, transitions take place, and the effect is that of a permanent dipole reorlenting in a fleld. This is a parelastic effect, represented by a relaxation process. The low frequency (high temperature) elastic constant is given by Eqs. 1-3, and the relaxation response for all frequencies by the Debye relaxation formulas

$$
(\Delta / \pi)=\Delta_{R}(\omega \tau) /\left[1+\omega^{2} \tau^{2}\right], \delta C / C=\Delta_{R} /\left[1+\omega^{2} \tau^{2}\right]
$$

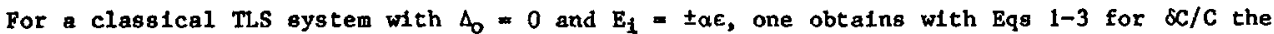
relaxation strength $\Delta_{R}$

$$
A_{R}=\delta C / C=\left(f \alpha^{2} / C k T\right) \operatorname{sech}^{2}(\alpha \varepsilon / k T)
$$

For $\varepsilon=0$, this reduces to $\mathrm{f} \alpha^{2} / \mathrm{CkT}$, the famillar classical Snoek peak result [10]. $\Delta_{R}$ is Inverse with temperature owing to the competing thermal disordering and external strain alligning effects. Also $\Delta_{\mathrm{R}}$ is proportional to the defect concentration and the square of the strain coupling constant $\alpha$, given by the slope of $\mathrm{E}(\varepsilon)$. The relaxation time is Arrehentus with an activation energy $U_{0} g$ iven by the height of the barrier. Transitions may occur by several processes, which act in parallel. Direct transition rates are independent of temperature at the lowest temperature and occur by tunneling, and increase linearly with temperature at high temperature where they become phonon-assisted. Indirect transitions through other states may also occur, but with a higher temperature dependence. The fastest rate dominates the temperature dependence. At high temperatures, in the classical regime, cransitions through states near the top of the barrier are the most effective.

The factor $\operatorname{sech}^{2}(\alpha \varepsilon / \mathrm{kT})$ acts as a cut-off at low temperatures. For carbon $\ln 1 \mathrm{ron}$, $\alpha-6 e v$. Using the values $\alpha \sim 5 \mathrm{eV}, \mathrm{kT} \sim 1 / 40 \mathrm{eV}$ at room temperature, one finds that $\operatorname{sech}^{2}(\alpha \varepsilon / \mathrm{kT})=0.98$ and 0.07 for $E=10^{-3}$ and $10^{-2}$ respectively. Such cut-off effects should thus be observable even in classical systems. For a distribution of strains, a sultable average over Eq. 7 should be taken. This has been done for a gaussian distribution of internal strains for the classical $E_{1}= \pm \alpha \varepsilon$ by Ish1guro, et al [11] In a study of the effect of uniaxial external static stress on the attenuation in p-type silicon.

An alternative and useful way of writing $\delta / C=\Delta_{8}+\Delta_{R}$, is, using

$$
\begin{gathered}
f_{1}=\left[f \exp \left(-E_{1} / k T\right)\right] / 2, \\
\Delta_{S}=\sum f_{1}\left(\partial^{2} E_{1} / \partial \varepsilon^{2}\right), \Delta_{R}=\sum\left(\partial E_{1} / \partial \varepsilon\right)\left(\partial E_{1} / \partial \varepsilon\right)
\end{gathered}
$$

This is the elastic constant change for thermal equilibrium, 1.e., assuming that there is sufficient time for the concentration $f_{1}$ to adjust to the thermal equilibrium value for each strain $\varepsilon$. The first term represents the elastic constant change owing to the curvature of the states for fixed concentration, and can be identified with the resonance $\Delta_{s}$. $\Delta_{s}=0$ for classical systems, where $\mathrm{B}$ is linear in $\varepsilon$. The second term represents the change resulting from the repopulation and can therefore be identified as the relaxation strength for a Debye relaxation process. 
For a quantum TLS, using $E_{1}= \pm \Delta$ of Eq. 4, one obtalns with Eqs. 1-3,

$$
\begin{gathered}
\Delta_{R}=\left(f \alpha^{2} / C k T\right)[\alpha \varepsilon / \Delta]^{2} \operatorname{sech}^{2}(\Delta / k T) \\
\Delta_{B}=\left(f \alpha^{2} / C \Delta_{b}\right)\left[\Delta_{o} / \Delta\right]^{3} \tanh (\Delta / k T)
\end{gathered}
$$

The new effects are $\Delta_{S}$ and the factor in square brackets in $\Delta_{R}$. Figs. 2 and 3 show results for $\Delta_{S}$ and $\Delta_{R}$ separately versus inverse temperature $\Delta_{0} / k T$ for varfous values of the normalized strain $B=\alpha \varepsilon / \Delta_{0}$, while Fig. 4 shows the result (dashed lines) for the total modulus change $\& / C$. The relaxation strengths have been normalized by $f \alpha^{2} / C A_{0}$. At high temperatures $\delta \mathrm{C} / \mathrm{C}=\mathrm{f} \alpha^{2} / \mathrm{CkT}$, 1ndependent of $\Delta_{0}$. At low temperatures $\Delta_{R}$ is cut-off to zero, while $\Delta_{S}$ achleves its maximum value. For small strain, $\Delta_{R}$ goes to zero as $(\alpha \varepsilon / \Delta)^{2}$ showing explicitly that symmetric TLS give no relaxation, while for large strain $\Delta_{S}$ goes to zero as $\left(\Delta_{0} / \Delta\right)^{3}$.

For a random strain distribution, $\Delta_{R}$ and $\Delta_{8}$ in Eqs. 10 and 11 may be averaged over a LorentLlan distribution [12]: $N(\varepsilon)=\varepsilon_{0} /\left[\pi\left(\varepsilon^{2}+\varepsilon_{0}^{2}\right)\right]$. Results for a random distribution are illustrated in Fig. 5 for $\beta=0.5$ and 2.0 , where now $\beta=\alpha \varepsilon_{0} / \Delta_{0}$. The figure shows $\Delta_{S}$ and the sum $\Delta_{S}+\Delta_{R}$ for two strains. Also shown is the sum for the delta function distribution of Fig. 4 for these values of strain. For the distribution, the low temperature cut-off 18 broadened for $\Delta_{R}$ and $\Delta_{S}$. The dependence of $\delta C / C$ at low temperature on strain is weakened because, even for large $\varepsilon_{0}$, some low strain systems are included in the distribution. This also accounts for the disappearance of a maximum in $6 \mathrm{C} / \mathrm{C}$ with temperature for large strains. At high temperature, the classical result for $\delta \mathrm{C} / \mathrm{C}$ is obtained, and at low temperatures an analytic result for $\delta \mathrm{C} / \mathrm{C}$ is avallable which permits a discussion of the dependence of the results on the gap [13]. These curves can be used in the analysis of data to obtain all the parameters of a TLS $\left(A_{0}, \alpha, \varepsilon_{0}\right)[14]$.

For a TLS, the one-phonon transition rate has the form

$$
T^{1}=A \Delta_{0}^{2} \Delta \operatorname{coth}(\Delta / k T)
$$

where $A$ is a constant [1]. At low temperatures $(T<<\Delta)$ this depends on $\Delta$ and therefore on straln. At high temperatures $(T\rangle\rangle), \tau-1=B T$ independent of $\Delta$ and therefore of stratn. Since the relaxation strength in Eq. 10 is zero for $\mathrm{T}<\Delta$, it is a good approximation to take $\mathrm{BT}$ for $T^{-1}$ in $\mathrm{Eq} .6$. Using $\mathrm{Eq} \cdot 10$, one then obtains

$$
\Delta / \pi=\Delta_{R}{ }^{-}(\varepsilon) /\left[1+\left(T / T_{m}\right)^{2}\right], \delta C / C=\Delta_{R}{ }^{-}\left(T / T_{m}\right) /\left[1+\left(T / T_{m}\right)^{2}\right]
$$

where $\Delta_{R}{ }^{\prime}(\varepsilon)=\left(f \alpha^{2} / C k T m\right)(\alpha \varepsilon / \Delta)^{2} \operatorname{sech}^{2}(\Delta / k T)$, and $T_{m}=\omega / B$. This 1s a Debye response, but the temperature dependence is inverted from the familtar form found for faster relaxation rates, with a maximum in the velocity change accompanied by a dispersion in the attenuation, all multiplied by the low temperature cut-off factor $\operatorname{sech}^{2}\left(\Delta / \mathrm{k}^{\mathrm{T}} \mathrm{T}\right)$. Since $\tau$ is independent of strain, the response remains of Debye form when Eq. 13 is averaged over a strain distribution, with only a new $\Delta_{R}\left(\varepsilon_{0}\right)$ defined. When the cut-off occurs while $\omega \tau \gg l$, there $1 s$ a region for which the attenuation is temperature independent while the elastic constant changes inearly with temperature. This may account for some unusual behavior found for dueterium trapped at oxygen in nioblum [15]. For a four-level system (FLS) with $E_{1,2}= \pm\left(\Delta_{0}^{2}+\alpha^{2} \varepsilon^{2}\right)^{1 / 2}$ and $E_{3}, .4= \pm \alpha \varepsilon$, one finds in the
same way

$$
\begin{gathered}
\Delta_{S}=\frac{1}{\left(1+\beta^{2}\right)^{3 / 2}}\left\{\frac{\sinh \left(1+\beta^{2} f^{/ 2} x\right.}{\cosh \beta x+\cosh \left(1+\beta^{2}\right)^{1 / 2} x}\right\} \\
A_{R}=-x\left\{\frac{\left\{\left(\beta /\left(1+\beta^{2}\right)^{1 / 2}\right] \sinh \left(1+\beta^{2}\right)^{1 / 2} x+\sinh \beta x\right.}{\cosh \left(1+\beta^{2}\right)^{1 / 2} x+\cosh \beta x}+x\left\{\frac{\left[\beta^{2} /\left(1+\beta^{2}\right)\right] \cosh (1+\beta)^{2 / 2} x+\cosh \beta x}{\cosh \left(1+\beta^{2}\right) x+\cosh x}\right\}\right.
\end{gathered}
$$

where $x=4_{0} / k T$.

The totel $\delta \mathrm{C} / \mathrm{C}=\Delta_{\mathrm{S}}+\Delta_{\mathrm{R}}$ 18 shown in Fig. 4 as the solid lines, compared to the dashed 1ines for a TLS with the same strains. The total FLS results are similar to those for TLS. This is to be expected since the high temperature ilmit is given by the classical result which is the same for both systems, and the low temperature limit is determined by the ground state, which is also the same for both systems. An important difference is that the FLS has a relaxation component even for small strains, because of the Iinear dependence on strain for the widdle two levels. These levels, however, are not coupled by phonon transitions to other levels, so that rates to achieve equilibrium for these states could be much lower than those connecting the outer states. In this case, one might observe effectively TLS behavior at low temperature. 


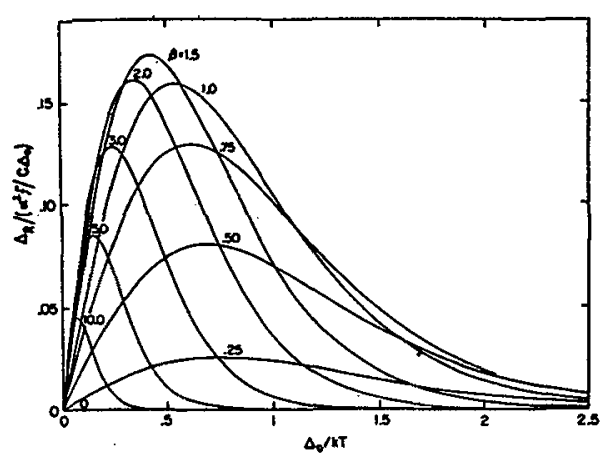

Fig. 2. Normalized relaxation strength as a function of normalized inverse temperature for various values of normalized strain.

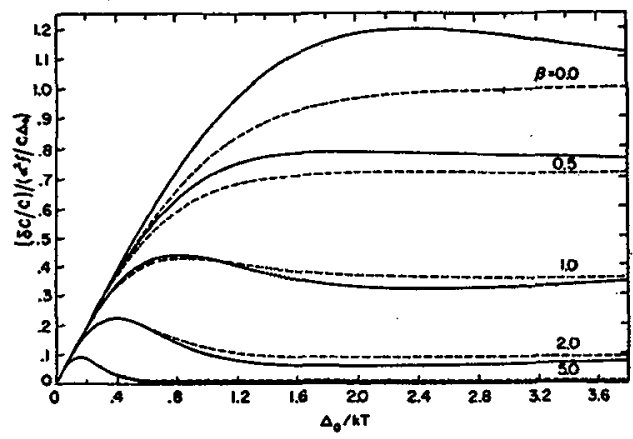

Fig. 4. Equilibrium relative modulus change (sum of relaxation and resonance strengths) as a funcrion of normalized inverse temperature for varlous values of norwalized strain. The solid (dashed) lines are for a FLE (TLS).

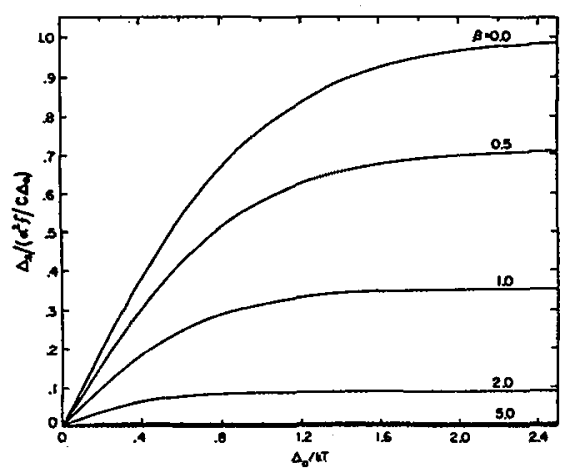

Fig. 3. Normalized resonance strength as a function of normalized inverse temperature for various values of normalized strain.

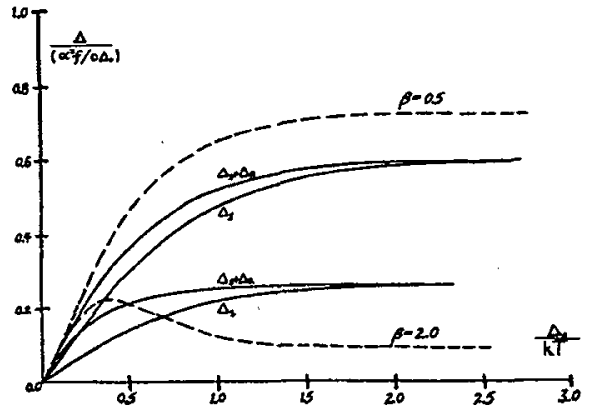

Fig - 5. Normalized resonance strengths $\Delta_{S}$ and total strength $\Delta_{S}+\Delta_{R}$ as a function of normalized inverse temperature for two values of normalized straln. The solld curves are for a random 8 train distribution and the dashed curves for delta function distribution.

\section{REFERENCES}

1. J. Jäckle, Z. Phys. 257, 212 (1972).

2. J. Jäckle, Z. Plché, W. Arnold and S. Hunklinger, J. Non-Cryst. Solids 20, 305 (1976).

3. J. L. Wang, G. Weiss, H. Wipf and A. Magerl, In Phonon Scattering in Condensed Matter, ed.

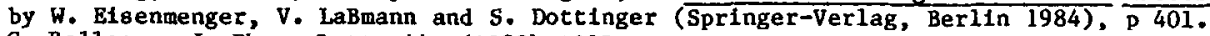

4. G. Bellessa, J. Phys. Lett. 44, (1983) 1387.

5. D. B. Poker, G. G. Setser, A. V. Granato, and H. K. Birnbaum, Phys. Rev. B29, 622 (1984).

6. B. Kroner, in Theory of Crystal Defects. ed, by B. Gruber, Acadenlc Press, N.Y. (1966), p. 215.

7. K. L. Hultman. Thesis, University fo Illinois (1979).

8. A. V. Granato, in Point Defect Interactions in Metals. ed by. J. J. Takamura, M. Dagama and M. Kiritani, Univ. of Tokyo Press (1982), p. 67.

9. Y. Kogure, W. K. B. Ho, and A. V. Granato, Ibid, p. 153.

10. A. S. Nowick and B. S. Berry, Anelastic Relaxation In Crystalline Solids, Academic Press, New York and London (1972).

11. T. Ishlguro, T. A. Pjeldly and C. Elbaum, Sol. State Com 10, 1309 (1972).

12. A. M. Stoneham, Rev Mod. Phys. 41, 82 (1969).

13. C. C. Yu and A. V. Granato, to be publiahed.

14. E. Drescher-Krasicka and A. V. Granato. (This conference).

15. K. F. Huang, Thes18, University of Illino1s (1982). 